\title{
Graphene spintronics: the role of ferromagnetic electrodes
}

\author{
Jesse Maassen,, , * Wei $\mathrm{Ji}^{2,}, 1$, ${ }^{\dagger}$ and Hong Guo ${ }^{1}$ \\ ${ }^{1}$ Centre for the Physics of Materials and Department of Physics, \\ McGill University, Montreal, QC, Canada, H3A $2 T 8$ \\ ${ }^{2}$ Department of Physics, Renmin University of China, Beijing 100872, China
}

\begin{abstract}
We report a first principles study of spin-transport under finite bias through a grapheneferromagnet $(\mathrm{FM})$ interface, where $\mathrm{FM}=\mathrm{Co}(111), \mathrm{Ni}(111)$. The use of Co and $\mathrm{Ni}$ electrodes achieves spin efficiencies reaching $80 \%$ and $60 \%$, respectively. This large spin filtering results from the materials specific interaction between graphene and the FM which destroys the linear dispersion relation of the graphene bands and leads to an opening of spin-dependent energy gaps of $\approx 0.4-0.5 \mathrm{eV}$ at the $\mathrm{K}$ points. The minority spin band gap resides higher in energy than the majority spin band gap located near $\mathrm{E}_{\mathrm{F}}$, a feature that results in large minority spin dominated currents.
\end{abstract}

The field of spintronics, or magneto-electronics, utilizes the spin degree of freedom of electrons and their inherent magnetic moment to influence or control the properties of a circuit. Within this field much effort has focused on developing interfaces, commonly a non-magnetic metal or insulator in contact with a ferromagnet (FM), that exhibits a large spin-polarized interface resistance [1]. Ideally, such a spin filter would allow electrons of only a single spin component to conduct.

Graphene, a 2D lattice of $\mathrm{C}$ atoms, is a gapless material with linear dispersion electronic bands joining at the Fermi level $\left(\mathrm{E}_{\mathrm{F}}\right)$ in conical (Dirac) points located at the $\mathrm{K}$ points in the Brillouin zone (BZ) 2]. It has received much attention due to it's exceptional properties [3], including zero effective mass carriers with extremely large mobilities, and is poised to play a role in the future of nanotechnology. Among other qualities, graphene has weak spin-orbit interaction due to the low atomic number of $\mathrm{C}$ resulting in long spin-flip scattering lengths. Hence, graphene is a promising material for applications in spintronic devices, where one can exploit graphene's unique electronic properties within the context of magnetoelectronics.

Generating and injecting a spin-polarized current into graphene is of vital importance to the development of graphene-based spintronics. Graphene nanoribbons, unlike pure graphene, are theoretically predicted to possess a local magnetic moment at the zigzag edges 4, but a major limitation arises in the difficulty of reliably fabricating well defined low-disorder edges. Thus, efficient spin injection into graphene is required for the realization of a prototypical spintronic device. A previous first principles study showed extremely large spin filtering efficiencies for $\mathrm{FM} \mid$ graphene $(\mathrm{Gr}) \mid \mathrm{FM}$ junctions $(\mathrm{FM}=\mathrm{Co}, \mathrm{Ni})[5$, in which the current flow was oriented perpendicular to the graphene. In this way, the spin-polarized current is primarily dominated by the inter-layer coupling, namely van der Waals interactions, between graphene sheets, rather than the characteristic graphene states. Moreover, the current-in-plane geometry, with transport occurring parallel to the graphene, is the most common experimental and theoretical device architecture [6] 9]. In such sys- tems the source and drain electrodes are comprised of FM-covered graphene (because metallic contacts are deposited on top of graphene) which, depending on the nature of the chemical bonding, can hybridize and result in a complex electronic structure. It was previously shown that graphene placed in contact with $\mathrm{Co}$ or $\mathrm{Ni}$ strongly hybridizes leading to significant modifications of the graphene bands [5. Demonstrated by a similar system [10, it can be inferred that the interaction between graphene and a FM is very sensitive to the particular atomic configuration at the interface of the two materials. Hence, it is crucial to properly characterize the detailed atomic structure, in order to obtain the correct electronic states at the interface and accurately analyze the spin-polarized transport properties of the device. Given the nature of this problem, one must employ atomistic $a b$ initio modeling for an accurate treatment of the chemical interaction at the contact.

A nano-structure was constructed to model the interface between a source or drain electrode (i.e., the FMcovered graphene) and a pure graphene channel, as shown in 1. This interface will be hereafter referred to as Gr|FM. This particular choice in system geometry was motivated by calculating interface properties that are independent of the device length. Thus, whether considering a very long (diffusive transport) or very short (ballistic transport) graphene channel, we expect the results presented in this work to remain valid at the interface. We consider both $\mathrm{Co}(111)$ and $\mathrm{Ni}(111)$ as the FM in contact with graphene, forming our electrodes. For graphenebased spintronics, $\mathrm{Co}(111)$ and $\mathrm{Ni}(111)$ are excellent candidates for FM contacts since their in-plane lattice constants nearly perfectly match that of graphene 5 , with experimental mismatch values of $1.8 \%$ (Co) and $1.3 \%$ $(\mathrm{Ni})$.

In this Letter, first principles density functional theory (DFT) total energy and non-equilibrium transport calculations were carried out to study the spin-polarized electronic structure and spin-dependent transport properties of $\mathrm{Gr} \mid \mathrm{FM}$ interfaces under finite bias. In particular, the atomic structure of the FM-covered graphene was fully relaxed by DFT total energy calculations. Given the 

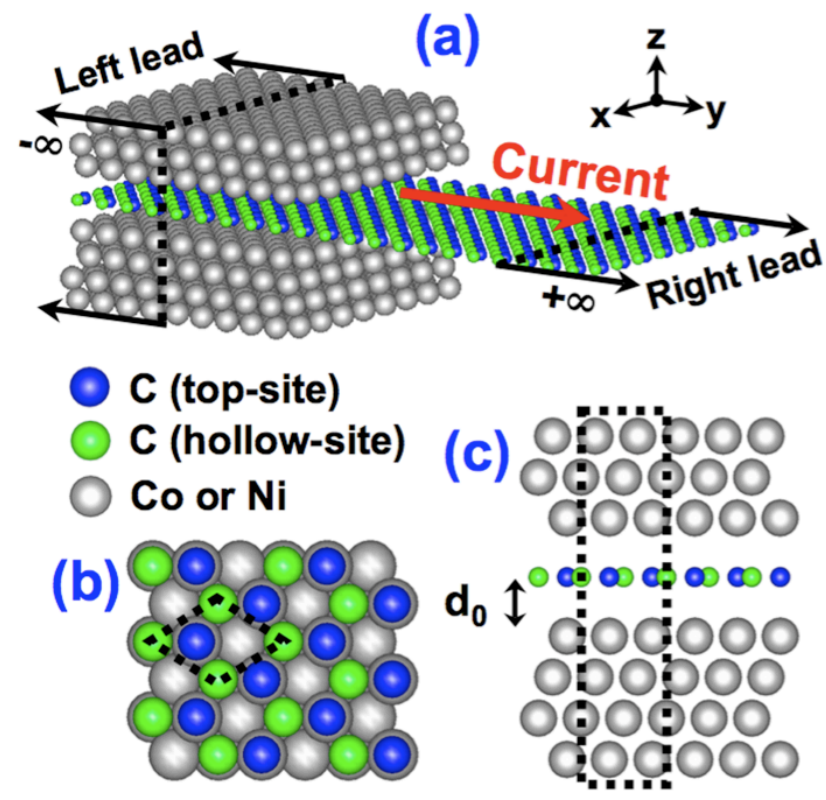

FIG. 1: (a) Diagram of the $\mathrm{Gr} \mid \mathrm{Co}(111)$ and $\mathrm{Gr} \mid \mathrm{Ni}(111)$ interface. The dotted lines indicate that the left and right leads, extending to $\pm \infty$, consist of FM-sandwiched graphene (left lead) and pure graphene (right lead). The top-site and hollowsite $\mathrm{C}$ atoms forming the graphene are shown in blue and green respectively. Periodic boundary conditions are assumed in the plane perpendicular to current, i.e., the system extends infinitely along the $\mathrm{x}$ - and $\mathrm{z}$-directions. (b) View of the left lead in the $\mathrm{x}-\mathrm{y}$ plane showing the graphene sitting on the FM. The top-site C is directly above the FM atom, while the hollow-site $\mathrm{C}$ is located at the hollow site. (c) View of the left lead in the $\mathrm{y}-\mathrm{z}$ plane indicating the optimized graphene-FM distance $d_{0}$. The dotted lines shown in (b) and (c) delimit the supercell box used for the electronic structure calculations.

optimized atomic coordinates, the spin-dependent band structure was analyzed. It was found that the strong hybridization between graphene and the FM destroys the linear dispersion relation of the graphene bands and opens spin-dependent band gaps at the K point (similar to previous work [5]). This FM-induced band gap opening of the graphene states results in spin-polarized currents that are minority (MIN) spin dominated due to the majority (MAJ) spin band gap residing near $\mathrm{E}_{\mathrm{F}}$. This electronic feature leads to spin filtering efficiencies reaching above $80 \%$ and $60 \%$ for $\mathrm{Gr} \mid \mathrm{Co}$ and $\mathrm{Gr} \mid \mathrm{Ni}$ interfaces, respectively. This work provides a unique analysis of the spin-polarized transport properties of a Gr|FM interface while considering the materials specific interactions in a non-equilibrium setting.

The structural relaxations and band structure calculations were performed using DFT, the local density approximation [11] for exchange-correlation potentials, the projector augmented wave method, and a plane wave basis with a cutoff energy of $400 \mathrm{eV}$ as implemented in the Vienna ab-initio simulation package [12]. 1(a) shows a diagram of the interface, where the left lead

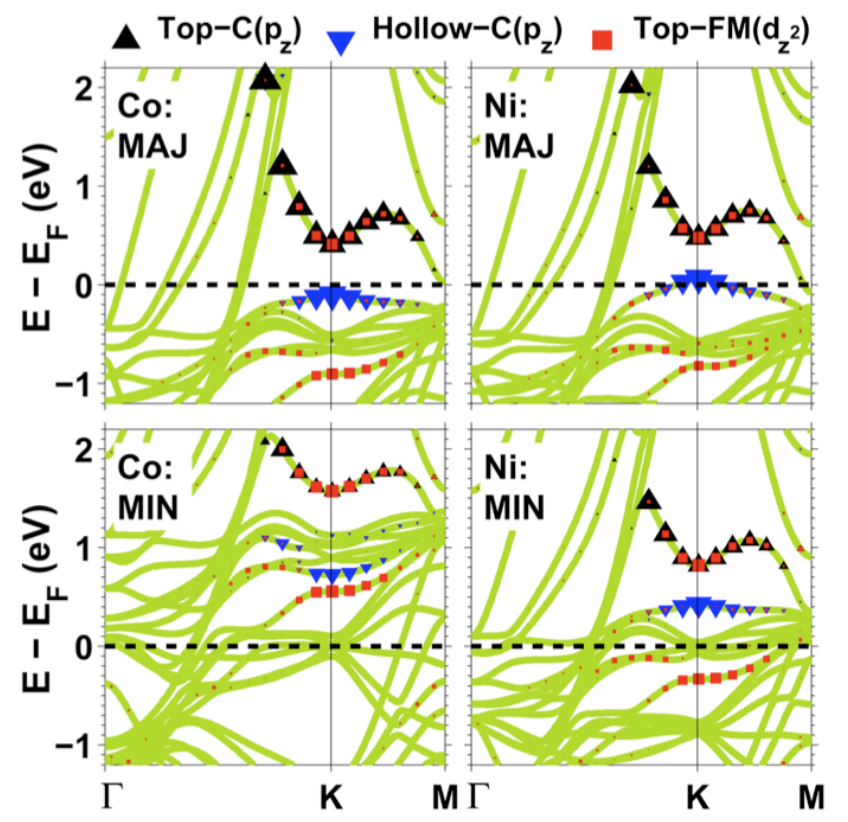

FIG. 2: Electronic structure of $\mathrm{Co}(111)$ - and $\mathrm{Ni}(111)$ sandwiched graphene (left lead of system in 1(a)). The left and right panels show the majority spin and minority spin bands for $\mathrm{Co}$ and $\mathrm{Ni}$ respectively. The Fermi level is indicated by the horizontal dashed line. The black up-triangles (blue down-triangles) show the $\mathrm{p}_{\mathrm{z}}$ character of the top-site (hollow-site) C, while the red diamonds present the $\mathrm{d}_{\mathrm{z}^{2}}$ character of the Co or $\mathrm{Ni}$ atoms located directly above and below the top-site C.

consists of FM-sandwiched graphene and the right lead is pure graphene. Transport occurs in the y-direction (as indicated by the red arrow) and periodic boundary conditions are assumed in the plane perpendicular to current, i.e., the system extends infinitely along the $\mathrm{x}$ - and z-directions. The most stable (minimal-energy) configuration for graphene on a $\mathrm{Co}(111)$ or $\mathrm{Ni}(111)$ substrate was used [5] and corresponds to the top-site $\mathrm{C}$ located directly above the FM atom and the hollow-site $\mathrm{C}$ sitting at the hollow site (see1(b) and 1(c)). Seven FM layers are used to separate the graphene sheets in adjacent supercells along the z-direction. The atomic structure of the graphene-FM contact was obtained by fixing the in-plane lattice constant to graphene's value of $2.46 \AA$ and relaxing the atoms in the supercell until the net forces acting on the atoms were below $0.01 \mathrm{eV} / \AA$. The supercell box height, in the direction perpendicular to graphene, was varied after each relaxation in order to find the optimal height through total energy minimization. A $k$-mesh of $21 \times 21 \times 3$ was adopted to sample the BZ for structural relaxations and total energy calculations. These parameters provide the optimal graphene-FM distance $d_{0}$ equal to $2.17 \AA$ and $2.13 \AA$ for $\mathrm{Gr} \mid \mathrm{Co}(111)$ and $\mathrm{Gr} \mid \mathrm{Ni}(111)$ respectively.

The spin-dependent band structure of the left lead is shown in 2. The green lines correspond to the bands of 
the hybrid graphene-FM system. To locate the states originating from graphene, the weight of the $\mathrm{C}\left(\mathrm{p}_{\mathrm{z}}\right)$ orbitals of all bands is superimposed (black up-triangle: top-site C, blue down-triangle: hollow-site C). There are three distinct features found from the figure: (i) At the $\mathrm{K}$ point, the graphene bands no longer show a linear dispersion relation and now exhibit a band gap opening of $\approx$ 0.4-0.5 eV, similar to what was shown for a single FM surface in contact with graphene [5]. The majority spin band gap resides at a lower energy than the minority spin band gap, and they do not overlap. This indicates that at specific energies, one finds electrons of only a single spin type in the graphene. (ii) Superimposing the $d_{z^{2}}$ character of the FM atoms located in the first layer above and below the graphene (plotted as red squares) shows that the FM interacts only via the top-site $\mathrm{C}$. This can be seen from the fact that the red squares only overlap with the black up-triangles. Note that only the $d_{z^{2}}$ orbitals showed any significant mixing with the graphene, in accordance with previous work [5]. (iii) It is also clear that the graphene conduction band (CB) and valence band (VB) result separately from the top-site $\mathrm{C}$ and the hollow-site $\mathrm{C}$, respectively. This is in contrast to pure graphene, where both $\mathrm{C}$ atoms in the primitive cell equally contribute to the $\mathrm{CB}$ and VB. The C-site dependence on the graphene $\mathrm{CB}$ and VB can be understood from the graphene-FM interaction (mentioned in (ii)) which breaks the sub-lattice symmetry between the top-site $\mathrm{C}$ and the hollow-site $\mathrm{C}$.

Given the fully relaxed atomic structures and the well understood electronic states of the left lead, we employed our state-of-the-art $a b$ initio transport package, named MATDCAL 13, to compute the spin-dependent transport properties of the whole junction. MATDCAL uses non-equilibrium Green's functions (NEGF) combined with DFT for open systems in a two-probe geometry under finite bias, where the leads extend to $\pm \infty$. An optimized double- $\zeta$ polarized atomic orbital set was built for each atomic species. The local density approximation [1] and norm-conserving non-local pseudopotentials [14] were used and $k$-point convergence tests, including the high symmetry points $\Gamma, \mathrm{K}$ and $\mathrm{M}$, were performed for all calculations.

The spin-dependent transmission coefficient of the minority states $\left(T_{M I N}\right)$ in the $2 \mathrm{D}$ BZ (in the plane of $k_{x}$ and $k_{z}$ which are $\perp$ to the transport direction, i.e., $\mathrm{y}$ direction) for $\operatorname{Gr}($ zigzag) $\mid \mathrm{Ni}$ is plotted in 3(a). The notation graphene(zigzag) or graphene(armchair) indicates the graphene is oriented such that transport occurs along the zigzag or armchair direction, respectively. $T_{M I N}$ shows a sharp peak at $k_{x}=0$, with near-zero values away from this point. To quantify this unusual dependence on $k$, we measure the width $\left(\delta k_{x}\right)$ of the $T_{M I N}$ peak. $\delta k_{x}$ is defined as the half-max width of $T$, as depicted in 3(a). 3(b) presents the calculated spin-polarized $\delta k_{x}$ as a function of energy $(E)$ for $\operatorname{Gr}$ (zigzag)|Ni, which clearly shows a nearly linear behavior. The spread in $\delta k_{x}$ can be traced
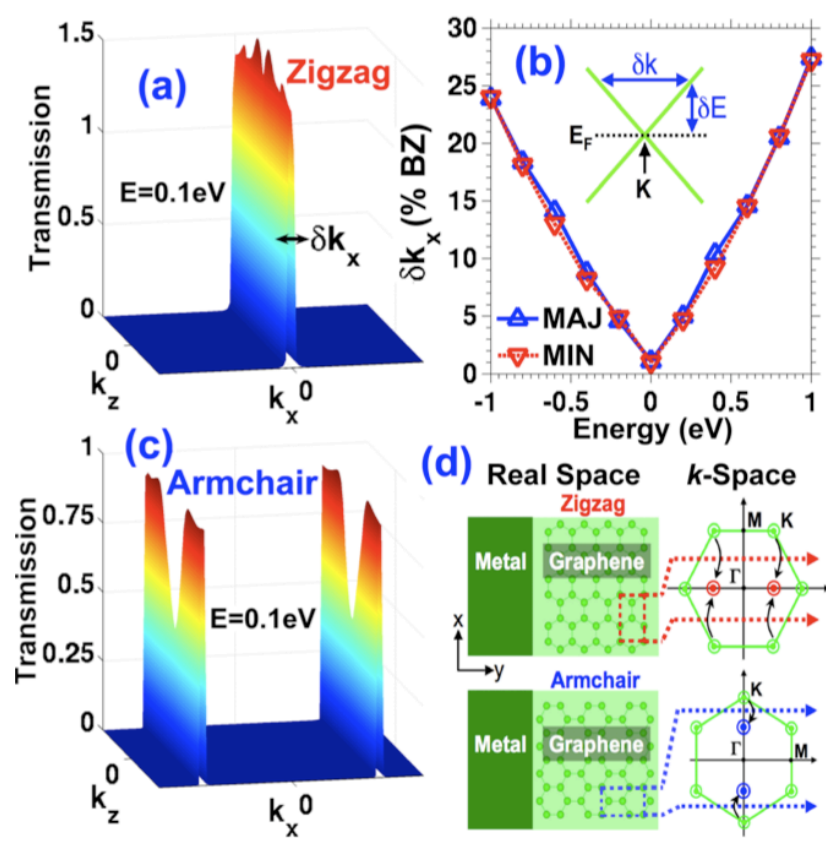

FIG. 3: (a) $T_{M I N}$ versus $k_{\perp}$ at $E=0.1 \mathrm{eV}$ for $\operatorname{Gr}($ zigzag) $\mid \mathrm{Ni}$. (b) Spin-polarized $\delta k_{x}$ versus $E$ for the same system as in (a). $\delta k_{x}$ is defined as the width at half-max of $T$. The inset illustrates the linear $E-k$ dispersion bands of graphene. (c) $T_{M I N}$ versus $k_{\perp}$ at $E=0.1 \mathrm{eV}$ for $\operatorname{Gr}$ (armchair) $\mid$ Co. (d) Left panels: Top view in real space of $\operatorname{Gr}($ zigzag) $\mid F M$ and $\operatorname{Gr}$ (armchair)|FM. Right panels: Primitive BZ of pure graphene(zigzag) (top) and graphene(armchair) (bottom). The dashed arrows indicate the transport direction as well as delimit the zone edges along $k_{x}$ for our calculations, due to the use of a non-primitive supercell (the supercell width along $\mathrm{x}$ is shown in the left insets). The black curved arrows illustrate the folding of the $\mathrm{K}$ points.

back to the conical states of pure graphene, which also exhibit a linear $E$ - $k$ relationship as shown in the inset of $3(\mathrm{~b})$. For a $\mathrm{Gr} \mid \mathrm{FM}$ interface, the pure graphene forming the right lead of our system only has electronic states at the $\mathrm{K}$ points (for $E \approx \mathrm{E}_{\mathrm{F}}$ ). Hence, all the incoming carriers originating from the FM-covered graphene (i.e., the source or drain electrode) are blocked by the pure graphene except those with states at the $\mathrm{K}$ points. 3(c) presents $T_{M I N}$ versus $k_{\perp}$ for $\operatorname{Gr}$ (armchair)|Co. The armchair-oriented graphene also shows sharp peaks along $k_{x}$, but are shifted in comparison to the zigzag-oriented graphene to $k_{x}= \pm 2 / 3$. The positions of the peaks along $k_{x}$ can be explained, for both graphene orientations, by considering the effect of band folding on the graphene states. In 3(d), we show the primitive BZ of graphene, where the dashed arrows indicate the transport direction in addition to delimiting the $k_{x}$ zone edges due to our non-primitive supercell. The $\mathrm{K}$ points located outside the $k_{x}$ zone edges will be folded inward, as illustrated with the black curved arrows. For graphene(zigzag), the conducting $\mathrm{K}$ points all appear at $k_{x}=0$ leading to a single peak in $T_{M I N}$, while the $\mathrm{K}$ points for graphene(armchair) 
are positioned at $k_{x}= \pm 2 / 3$, as seen in 3(a) and 3(c).

The $k$-averaged spin-dependent transmission coefficients $(T)$ versus energy $E$ at equilibrium (zero bias) for $\mathrm{Gr}$ (zigzag) $\mid \mathrm{Co}(\mathrm{Ni})$ are presented in $4(\mathrm{a})$ and 4(b), which were calculated using $T_{\sigma}(E)=$ $1 / A_{B Z} \int_{B Z} T_{\sigma}\left(E, k_{\perp}\right) d k_{\perp}$, where $A_{B Z}$ is the area of the BZ. For $E$ smaller than $\approx \mathrm{E}_{\mathrm{F}}\left(\mathrm{E}_{\mathrm{F}}\right.$ is set to zero), it is found that both $T_{M A J}$ and $T_{M I N}$ vary roughly linearly, similar to pure graphene. However, the $T$ values for $\mathrm{Gr} \mid \mathrm{FM}$ are roughly $50 \%$ smaller than those of pure graphene, a result of the band gap opening which reduces the band velocity. When considering $E>\mathrm{E}_{\mathrm{F}}$, one noticeable feature for both $\mathrm{Gr} \mid \mathrm{Co}$ and $\mathrm{Gr} \mid \mathrm{Ni}$ is the small $T_{M A J}$ value between $E \approx 0-0.4 \mathrm{eV}$. This energy range corresponds to the majority spin band gap of the graphene states (see 2), thus explaining the decrease in $T_{M A J}$. A similar effect occurs for $\mathrm{Gr} \mid \mathrm{Ni}$ but with $T_{M I N}$ (instead of $T_{M A J}$ ) between $0.43-0.80 \mathrm{eV}$, an energy range which overlaps with the minority spin band gap. 4(e) presents the spin transmission ratio $(\gamma)$ defined as $T_{\sigma} / T_{\bar{\sigma}}$, where $\sigma$ is the spin component with the larger $T$ value and $\bar{\sigma}$ is the opposite spin. $\gamma$ is defined positive (negative) when $\sigma$ is the majority (minority) spin. $\mathrm{Gr} \mid \mathrm{Co}$ and $\mathrm{Gr} \mid \mathrm{Ni}$ both yield large dips near $-0.1 \mathrm{eV}$ and $0.1 \mathrm{eV}$ with $\gamma$ approaching 18 and 25 respectively, each coinciding with the smallest $T_{M A J}$ value. Interestingly, for $\mathrm{Gr} \mid \mathrm{Ni}, \gamma$ shifts from minority spin dominated at $0.1 \mathrm{eV}$ to majority spin dominated at $0.5 \mathrm{eV}$. This crossover behavior is attributed to the end of the majority spin band gap and the beginning of the minority spin band gap both located near $0.4 \mathrm{eV}$.

The non-equilibrium calculations reveal the spinpolarized current $\left(I_{\sigma}\right)$-voltage $(V)$ characteristics of the $\mathrm{Gr} \mid \mathrm{Co}$ and $\mathrm{Gr} \mid \mathrm{Ni}$ interfaces, as plotted in 4(c) and (d). $I_{\sigma}$ is obtained from

$$
I_{\sigma}=\frac{e}{h} \int_{-\infty}^{\infty} T_{\sigma}(E)\left[f_{L}\left(E, \mu_{L}\right)-f_{R}\left(E, \mu_{R}\right)\right] d E,
$$

where $e$ is the electron charge, $h$ is Planck's constant and $f(E, \mu)$ is the Fermi-Dirac distribution. An applied bias $V$ varies the left and right chemical potentials as $\mu_{L}=E_{F}$ and $\mu_{R}=E_{F}+|e| V$, where $V=V_{L}-V_{R}$. For $\mathrm{Gr} \mid \mathrm{Co}$, it was found that $\left|I_{M I N}\right|>\left|I_{M A J}\right|$ for all $V$ values in the bias voltage window of interest. Whereas in the case of $\mathrm{Gr}|\mathrm{Ni},| I_{M I N}|>| I_{M A J} \mid$ is only observed for positive $V$ while the opposite result is found for negative $V$ (although less pronounced). To illustrate this, we plot the spin efficiency $(\eta)$ for the $\mathrm{Gr} \mid \mathrm{FM}$ interfaces in $4(\mathrm{f}) . \eta$ is calculated using $\left|I_{\sigma}-I_{\bar{\sigma}}\right| /\left|I_{\sigma}+I_{\bar{\sigma}}\right|$, where $\eta$ is defined positive (negative) when $\left|I_{M A J}\right|>\left|I_{M I N}\right|$ $\left(\left|I_{M I N}\right|>\left|I_{M A J}\right|\right)$. $\mathrm{Gr} \mid \mathrm{Co}$ and $\mathrm{Gr} \mid \mathrm{Ni}$ achieve maximal spin efficiencies above $80 \%$ (at $-0.2 \mathrm{~V}$ ) and $60 \%$ (at $0.4 \mathrm{~V}$ ) respectively, representing the percentage of net spin-polarized current. $\eta \rightarrow 0$ for negative $V$ in the case of $\mathrm{Gr} \mid \mathrm{Ni}$. This occurs because the integration window of $E$ for calculating $I_{\sigma}$ (ranging from $\left(\mathrm{E}_{\mathrm{F}}+|e| V\right) \rightarrow \mathrm{E}_{\mathrm{F}}$, valid for $V<0$ at zero temperature) is located below

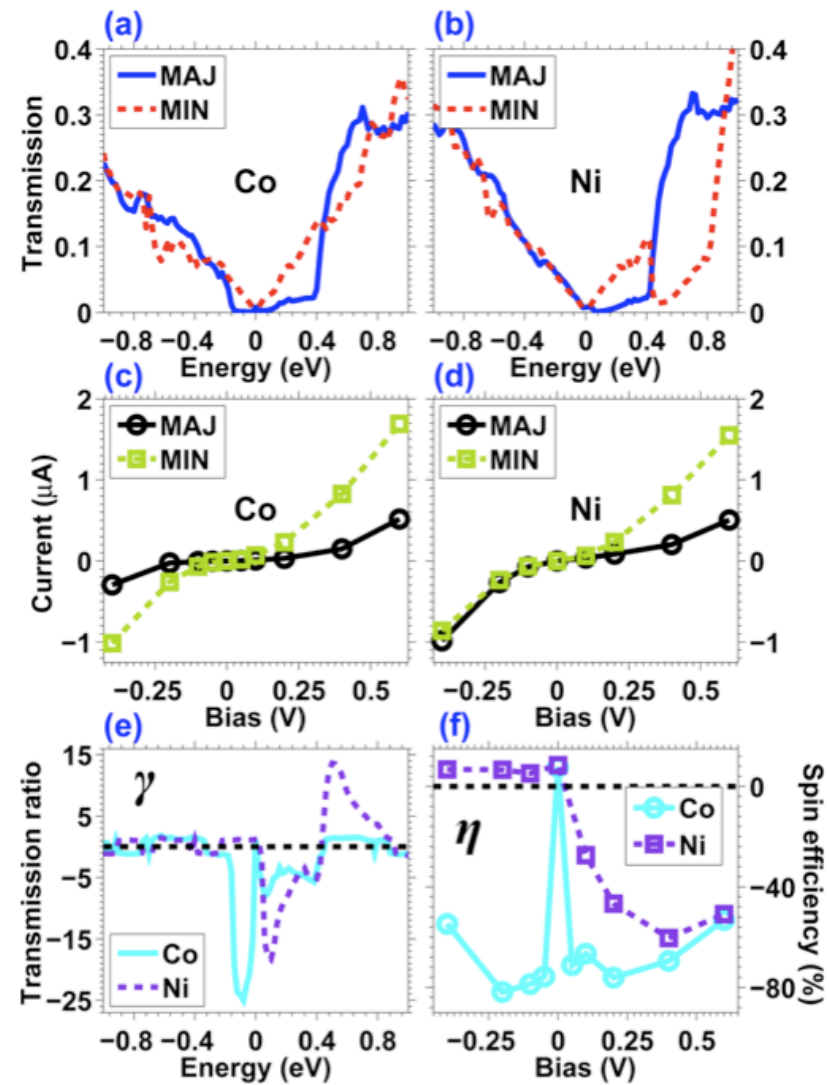

FIG. 4: (a)-(b): Spin-polarized $T$ versus $E$ at $V=0$ for $\mathrm{Gr} \mid \mathrm{Co}$ (a) and $\mathrm{Gr} \mid \mathrm{Ni}$ (b) (majority spin: solid blue line, minority spin: dashed red line). The Fermi level is set to $E=0$. (c)-(d): Spin-polarized $I$ versus $V$ for $\mathrm{Gr} \mid \mathrm{Co}$ (c) and $\mathrm{Gr} \mid \mathrm{Ni}$ (d) (majority spin: black circles, minority spin: green squares). (e) Transmission ratio $\gamma \equiv T_{\sigma} / T_{\bar{\sigma}}$ (where $\sigma$ is the spin component with the largest $T$, and $\bar{\sigma}$ is the opposite spin) versus $E$ for $\mathrm{Gr} \mid \mathrm{Co}$ (cyan full line) and $\mathrm{Gr} \mid \mathrm{Ni}$ (purple dashed line). Note that $T_{\sigma} / T_{\bar{\sigma}}$ is defined positive (negative) when $T_{M A J}>T_{M I N}\left(T_{M I N}>T_{M A J}\right)$. (f) Spin efficiency $\eta \equiv\left|I_{\sigma}-I_{\bar{\sigma}}\right| /\left|I_{\sigma}+I_{\bar{\sigma}}\right|$ versus $V$ for $\mathrm{Gr} \mid \mathrm{Co}$ (cyan circles) and $\mathrm{Gr} \mid \mathrm{Ni}$ (purple squares). Spin efficiency is defined positive (negative) when $\left|I_{M A J}\right|>\left|I_{M I N}\right|\left(\left|I_{M I N}>I_{M A J}\right|\right)$.

both majority and minority spin band gaps (see 2). This results in near-equal currents from both spin types. For Gr|Co, $\eta$ saturates slowly to zero with decreasing $V$, because when the integration range extends to the bottom of the majority spin band gap, located near $-0.15 \mathrm{eV}$, $T_{M A J}$ remains small due to the vanishing density of states at the Dirac point in the pure graphene (which is pinned at the lower boundary of the integration window). Hence, $V$ must decrease beyond $-0.15 \mathrm{~V}$ to obtain $\eta \rightarrow 0$. The peak at $V=0$ results from $I_{M A J}$ and $I_{M I N}$ both vanishing in pure graphene. The results shown in 4 have considered only graphene(zigzag). The transport properties of graphene(armchair) are found to be qualitatively similar to graphene(zigzag).

In summary, our non-equilibrium first principles transport calculations showed that $\mathrm{Gr} \mid \mathrm{Co}(111)$ and $\mathrm{Gr} \mid \mathrm{Ni}(111)$ 
interfaces exhibit large spin injection values reaching $80 \%$ and $60 \%$ respectively. This effect originates from the graphene-FM hybridization which leads to an opening of the conical Dirac bands resulting in spin-dependent energy gaps of the graphene states. Thus, in the ballistic regime, one can (theoretically) exploit the materials specific bonding between graphene and the FM to achieve very efficient spin filtering. However, it remains to be shown whether these spin properties are robust in the inevitable presence of random interface disorder.

Acknowledgements. This work was supported by the FQRNT of Quebec, NSERC of Canada and CIFAR. Calculations were performed using the RQCHP supercomputers.

* Electronic address: maassenj@physics.mcgill.ca

† Electronic address: wji@ruc.edu.cn

[1] Žutić, I.; Fabian, J.; Sarma, S. D. Rev. Mod. Phys. 2004, $76,323$.

[2] Castro Neto, A. H.; Guinea, F.; Peres, N. M. R.; Novoselov, K. S.; Geim, A. K. Rev. Mod. Phys. 2009, 81, 109.

[3] Geim, A. K.; Novoselov, K. S. Nature Materials 2007, 6, 183.

[4] Son, Y. W.; Cohen, M. L.; Louie, S. G. Nature 2006, 444, 347-349.

[5] Karpan, V. M.; Giovannetti, G.; Khomyakov, P. A.; Talanana, M.; Starikov, A. A.; Zwierzycki, M.; van den Brink, J.; Brocks, G.; Kelly, P. J. Phys. Rev. Lett. 2007, 99, 176602; Karpan, V. M.; Khomyakov, P. A.; Starikov, A. A.; Giovannetti, G.; Zwierzycki, M.; Talanana, M.;
Brocks, G.; van den Brink, J.; Kelly, P. J. Phys. Rev. B 2008, 78, 195419.

[6] Hill, E. W.; Geim, A. K.; Novoselov, K.; Schedin, F.; Blake, P. IEEE Trans. Magn. 2006, 42, 2694; Tombros, N.; Jozsa, C.; Popincius, M.; Jonkman, H. T.; van Wees, B. J. Nature 2007, 448, 571; Cho, S.; Chen, Y.-F.; Fuhrer, M. S. Appl. Phys. Lett. 2007, 91, 123105; Han, W.; Pi, K.; Bao, W.; McCreary, K. M.; Li, Y.; Wang, W. H.; Lau, C. N.; Kawakami, R. K. Appl. Phys. Lett. 2009, 94, 222109.

[7] Yokoyama, T. Phys. Rev. B 2008, 77, 073413; Ding, K.H.; Zhu, Z.-G.; Berakdar, J. Phys. Rev. B 2009, 79, 045405; Zou, J.; Jin, G.; Ma, Y.-q. J. Phys.:Condens. Matter 2009, 21, 126001; Dell'Anna, L.; De Martino, A. Phys. Rev. B 2009, 80, 155416; Krompiewski, S. Phys. Rev. B 2009, 80, 075433; Honda, S.; Yamamura, A.; Hiraiwa, T.; Sato, R.; Inoue, J.; Itoh, H. Phys. Rev. B 2010, 82, 033402.

[8] Kim, W. Y.; Kim, K. S. Nature Nanotech. 2008, 3, 408; Sahin, H.; Senger, R. T. Phys. Rev. B 2008, 78, 205423; Ozaki, T.; Nishio, K.; Weng, H.; Kino, H. Phys. Rev. B 2010, 81, 075422.

[9] Maassen, J.; Ji, W.; Guo, H. arXiv:1009.1066; accepted in Applied Physics Letters.

[10] Ning, Z.; Ji, W.; Guo, H. arXiv:090\%.4674 (unpublished).

[11] Perdew, J. P.; Zunger, A. Phys. Rev. B 1981, 23, 5048.

[12] Kresse, G.; Furthmuller, J. Phys. Rev. B 1996, 54, 11169; Comput. Mater. Sci. 1996, 6, 15.

[13] Taylor, J.; Guo, H.; Wang, J. Phys. Rev. B 2001, 63, 245407; 2001, 63, 121104(R); Waldron, D.; Haney, P.; Larade, B.; MacDonald, A.; Guo, H. Phys. Rev. Lett. 2006, 96, 166804; Maassen, J.; Zahid, F.; Guo, H. Phys. Rev. B 2009, 80, 125423.

[14] Troullier, N.; Martins, J. L. Phys. Rev. B 1991, 43, 1993. 